\title{
Phase transitions in nanoconfined binary mixtures of highly oriented colloidal rods
}

\author{
Daniel de las Heras* \\ Departamento de Física Teórica de la Materia Condensada, \\ Universidad Autónoma de Madrid, E-28049 Madrid, Spain \\ Yuri Martínez-Ratón† \\ Grupo Interdisciplinar de Sistemas Complejos (GISC), Departamento de Matemáticas, \\ Escuela Politécnica Superior, Universidad Carlos III de Madrid, \\ Avenida de la Universidad 30, E-28911, Leganés, Madrid, Spain \\ Enrique Velascd \\ Departamento de Fúsica Teórica de la Materia Condensada \\ and Instituto de Ciencia de Materiales Nicolás Cabrera, \\ Universidad Autónoma de Madrid, E-28049 Madrid, Spain
}

(Dated: April 18, 2022) 


\begin{abstract}
We analyse a binary mixture of colloidal parallel hard cylindrical particles with identical diameters but dissimilar lengths $L_{1}$ and $L_{2}$, with $s=L_{2} / L_{1}=3$, confined by two parallel hard walls in a planar slit-pore geometry, using a fundamental-measure density functional theory. This model presents (arXiv:1002.0612v, accepted in Phys. Rev. E) nematic (N) and two types of smectic (S) phases, with first- and second-order N-S bulk transitions and S-S demixing, and surface behaviour at a single hard wall which includes complete wetting by the $\mathrm{S}$ phase mediated (or not) by an infinite number of surface-induced layering (SIL) transitions. In the present paper the effects of confinement on this model colloidal fluid mixture are studied. Confinement brings about profound changes in the phase diagram, resulting from competition between the three relevant length scales: pore width $h$, smectic period $d$ and length ratio $s$. Four main effects are identified: (i) Second-order bulk N-S transitions are suppressed. (ii) Demixing transitions are weakly affected, with small shifts in the $\mu_{1}-\mu_{2}$ (chemical potentials) plane. (iii) Confinement-induced layering (CIL) transitions occurring in the two confined one-component fluids in some cases merge with the demixing transition. (iv) Surface-induced layering (SIL) transitions occurring at a single surface as coexistence conditions are approached are also shifted in the confined fluid. Trends with pore size are analysed by means of complete $\mu_{1}-\mu_{2}$ and $p-\bar{x}$ (pressure-mean pore composition) phase diagrams for particular values of pore size. This work, which is the first one to address the behaviour of liquid-crystalline mixtures under confinement, could be relevant as a first step to understand self-assembling properties of mixtures of metallic nanoparticles under external fields in restricted geometry.
\end{abstract}

PACS numbers:

*Electronic address: daniel.delasheras@uam.es

${ }^{\dagger}$ Electronic address: yuri@math.uc3m.es

†Electronic address: enrique.velasco@uam.es 


\section{INTRODUCTION}

Understanding the self-assembly of nanowires and metallic or semiconducting nanorods will be crucial in next-generation nanoelectronic and display-technology applications [1]. These technologies exploit the ordering of nanorods to create well-ordered arrays for the selective fabrication of devices. Inorganic nanoparticles with controlled size, shape and composition, exhibiting interesting optical, magnetic and electric properties are now being synthetised [2, 3]. But most applications are based on particle arrangements in large structures. Confinement of the rods in nanoscale geometries may be one possible strategy to create such structures. External electric or magnetic field can be used to orient particles in the required directions [4]. Predicting the shape and composition effects that lead to ordering in collections of nanometer-sized particles in a solvent is a theoretical challenge, and understanding entropic effects associated with the rodlike shapes is a prerequisite before considering other interparticle forces. Therefore, the interest in hard-particle models and in the statistical mechanics of positional and orientational ordering in fluids of identical or polydisperse hard particles has been revitalised in recent years.

The conceptual aspects of freezing of monodisperse particles in nanopores has been studied quite extensively [5-16]. On the other hand, smectic and layered phases made of anisometric particles present partial (one-dimensional) ordering and give an opportunity to study partial, as opposed to complete, spatial order in confined geometries [17]. Frustration effects in mesophases, normally associated with antagonistic conditions imposed on the nematic director in the form of surface and bulk fields, are crucial in technological applications of these materials [18]. But in smectic materials another type of frustration occurs, since the natural layer spacing $d$ establishes a length scale which will compete with any other length scale applied in the form of geometric constraints. The simplest confinement geometry is the planar slit pore, where the material is sandwiched between two parallel planar substrates. The confinement of two- or three-dimensional one-component fluids in the smectic or lamellar regime leads to complex behaviour [19 21] induced by commensuration effects between the pore width $h$ and the smectic spacing $d$. Theoretical work indicates that the possible phase behaviour includes confinement-induced layering (CIL) transitions, as well as a 'wavy' nematic-smectic transition strongly coupled to the latter. The strong smectic regime has not been studied experimentally yet. 
In contrast to the case of pure materials, confined mixtures of liquid-crystal forming particles have been given no attention. In our previous work [22], we have analysed the bulk and adsorption properties of a mixture of hard cylinders. A complex bulk phase diagram resulted, with nematic $(\mathrm{N})$ and smectic $(\mathrm{S})$ phases. The smectic phases came in two versions. In the first, non-microsegregated, smectic phase $\left(\mathrm{S}_{1}\right)$, layers are identical and composed of a mixture, in various proportions, of the two species. In the second, or microsegregated smectic phase $\left(\mathrm{S}_{2}\right)$, layers rich in one species alternate with layers rich in the other. Various phase transitions, including first- and second-order N-S transitions, as well as smectic demixing, were obtained; see Fig. 2(a), where the bulk phase diagram is depicted in the plane pressure $p$ vs. composition $x$ (defined as mole fraction of the short particles). The adsorption properties of the mixture on a hard wall were also studied in [22]. We found a complex behaviour, with the wall inducing strong layering leading to stratification of the material into alternating layers rich in one of the components. The surface behaviour includes complete wetting by the S phase, mediated (or not) by an infinite number of surface-induced layering (SIL) transitions, as well as critical adsorption on approaching second-order N-S transitions. As interactions between particles and wall are of hard-core type, layering and wetting properties of the nematic fluid in contact with the wall have an entropic origin. Similar phenomena have been found in theoretical work describing the surface phase behaviour of colloid-polymer mixtures [23].

In the present paper we extend the analysis of this mixture by considering fluids confined by two identical hard walls. This is a novel system where a new length scale appears: apart from the pore width $h$, there are two particle lengths, $L_{1}$ and $L_{2}$. Commensuration between the three length scales brings about new phenomena. The most salient results of our work are: (i) Second-order bulk N-S transitions are suppressed. (ii) Demixing transitions are not much affected by confinement, with small shifts in the $\mu_{1}-\mu_{2}$ (chemical potentials) or $p-\bar{x}$ (pressure vs. mean pore composition) planes. (iii) CIL transitions occurring in the one-component fluids are modified by mixing, ending in critical points or at the demixing transition. (iv) SIL transitions on each wall are also modified by the confinement; the very first transitions (the lowest-order ones, involving the first few layers) may survive in wide pores, while the others are preempted by demixing; in some cases some of them may reappear in the demixing region creating islands of stability, while higher-order SIL transitions are suppressed. In general, both types of layering transitions tend to disappear as the pore 
width is decreased. (v) Some new phenomena, genuinely connected to confinement of the mixture, i.e. to the competition between the three length scales, $L_{1}, L_{2}$ and $h$, may also arise.

After summarising the density-functional model used in the analysis and discussing briefly the bulk and surface phase behaviour in the next section, we present in Section III the results for the confined mixture, considering wide and narrow slit pores. Finally, some conclusions are presented in Section IV]

\section{MODEL, THEORY AND BULK AND SURFACE PHASE DIAGRAMS}

\section{A. Model}

We consider a binary mixture of parallel hard cylinders, with lengths $L_{1}, L_{2}$ and identical diameters $D$; see Fig. 1(a). Label 1 refers to the species of the short cylinders. We have selected $L_{1}$ as the unit of length. The length ratio investigated is $s=L_{2} / L_{1}=3$. The diameter $D$ is adjusted so that the transverse particle area in units of $L_{1}$ is set to unity, i.e. $\pi D^{2} / 4 L_{1}^{2}=1$. Since particles are parallel, bulk properties only depend on $s$, and the particular values of $L_{1}$ (or $L_{2}$ ), and $D$ are irrelevant. As discussed in [22], the values of particle lengths chosen gives a binary mixture that would be equivalent to a mixture of freely-rotating but highly oriented cylinders (of the same length ratio) such that both components would have a stable smectic phase. The cylinders are in numbers $N_{1}$ and $N_{2}$, so that the composition of the mixture is defined as $x=N_{1} / N$, with $N=N_{1}+N_{2}$ the total number of particles. The cylinder axes are chosen to lie along the $z$ direction; see Fig. 1(b). This configuration models a real colloidal or molecular fluid where, either by surface treatment or by means of a bulk field, particles are forced to point along some fixed direction which, in the case of an adsorption system, would be the surface normal.

\section{B. Theory}

The properties of the mixture are analysed using a fundamental-measure version of density-functional theory [24, 25]. This theory is in very good agreement with Monte Carlo simulations of monodisperse (one-component) cylinders, and there are no reasons to believe that the theory is not equally accurate for mixtures. We choose the grand potential $\Omega$ as the 
(a)

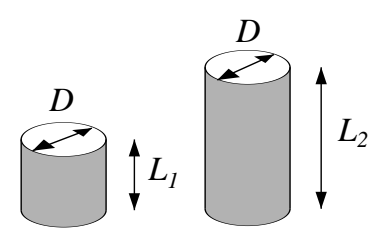

(b)

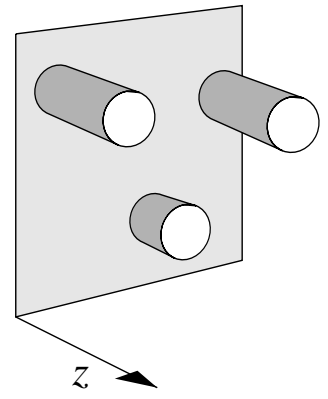

FIG. 1: (a) Schematic of particles studied. (b) Geometry of the adsorbed and confined fluid.

relevant thermodynamic potential. If $\rho_{i}(\boldsymbol{r})$ is the local number density distribution of the cylinder centres of mass of the $i$-th species, the grand-potential functional of the confined mixture is

$$
\Omega\left[\left\{\rho_{i}\right\}\right]=\mathcal{F}\left[\left\{\rho_{i}\right\}\right]-\sum_{i=1}^{2} \mu_{i} \int_{V} d \boldsymbol{r} \rho_{i}(\boldsymbol{r}),
$$

where $\mu_{i}$ is the chemical potential of the $i$-th species. The Helmholtz free-energy functional $\mathcal{F}\left[\left\{\rho_{i}\right\}\right]$ is written, as usual [22], as

$$
\mathcal{F}\left[\left\{\rho_{i}\right\}\right]=\mathcal{F}_{\text {id }}\left[\left\{\rho_{i}\right\}\right]+\mathcal{F}_{\text {ex }}\left[\left\{\rho_{i}\right\}\right]+\mathcal{F}_{\text {ext }}\left[\left\{\rho_{i}\right\}\right] .
$$

$\mathcal{F}$ consists of three parts: the ideal-gas part,

$$
\mathcal{F}_{\text {id }}\left[\left\{\rho_{i}\right\}\right]=k T \sum_{i=1}^{2} \int_{V} d \boldsymbol{r} \rho_{i}(\boldsymbol{r})\left\{\log \left[\mathcal{V}_{i} \rho_{i}(\boldsymbol{r})\right]-1\right\},
$$

the excess part,

$$
\mathcal{F}_{\text {ex }}\left[\left\{\rho_{i}\right\}\right]=k T \int_{V} d \boldsymbol{r} \Phi\left(\left\{\rho_{i}(\boldsymbol{r})\right\}\right),
$$

and the external contribution from the walls:

$$
\mathcal{F}_{\text {ext }}\left[\left\{\rho_{i}\right\}\right]=k T \sum_{i=1}^{2} \int_{V} d \boldsymbol{r} \rho_{i}(\boldsymbol{r}) V_{\text {ext }}^{(i)}(\boldsymbol{r}) .
$$

Note that temperature $T$ is not a relevant variable since all interactions are purely hard and therefore $\mathcal{F}$ is proportional to $T$. In the above $k$ is Boltzmann constant, $\mathcal{V}_{i}$ is the thermal volume of species $i$, and $\Phi\left(\left\{\rho_{i}(\boldsymbol{r})\right\}\right)$ is the excess free-energy density. In the fundamentalmeasure approximation [24, 25], $\Phi(\mathbf{r})$ depends on the local averaged one-body $n_{i}(\boldsymbol{r})$ and twobody weighted densities $N_{i}(\mathbf{r})$. However, by imposing the density profiles to be functions only 
on $z$ (smectic symmetry), these weighted densities reduce to only two (one-body) densities, $n(z)$ and $\eta(z)$. In this case the expression for the free-energy density is given by

$$
\Phi=n\left\{-\ln (1-\eta)+\frac{3 \eta}{1-\eta}+\frac{\eta^{2}}{(1-\eta)^{2}}\right\} .
$$

The averaged densities are

$$
n(z)=\frac{1}{2} \sum_{i=1}^{2}\left[\rho_{i}\left(z-\frac{L_{i}}{2}\right)+\rho_{i}\left(z+\frac{L_{i}}{2}\right)\right], \quad \eta(z)=a_{0} \sum_{i=1}^{2} \int_{z-L_{i} / 2}^{z+L_{i} / 2} \rho_{i}\left(z^{\prime}\right) d z^{\prime},
$$

where $a_{0}=\pi D^{2} / 4$ is the transverse area of the cylinder. $\eta(z)$ is the local packing fraction.

The procedure to obtain the expression for $\Phi(\mathbf{r})$ consists of the following two steps: (i) a density functional is derived for a mixture of two-dimensional hard disks, with the important property of dimensional cross-over from two to one dimension (i.e. when disks are located on a straight line, the functional should reduce to the exact one for a mixture of hard segments). (ii) If we impose the parallel-alignment constraint on particles, the density functional for a mixture of parallel hard cylinders can be obtained from the density functional for the hard-disk mixture just derived by applying again the dimensional cross-over criterion. This is easy to visualize by taking into account that the projections of the cylinders on a plane perpendicular to their axes just give a mixture of disks with different diameters [24].

In the interfacial problems to be studied below, inhomogeneities resulting from the presence of surfaces will be included via an external potential, which consists of hard walls:

$$
V_{\text {ext }}^{(i)}(\boldsymbol{r})=\left\{\begin{array}{l}
\infty, z<\frac{L_{i}}{2} \text { or } z>h-\frac{L_{i}}{2}, \\
0, \quad \frac{L_{i}}{2} \leq z \leq h-\frac{L_{i}}{2},
\end{array} i=1,2,\right.
$$

where $h$ is the width of the slit pore. The conditions on the confined fluid are controlled by the chemical potentials $\mu_{1}$ and $\mu_{2}$ of the bulk phase with which it is in equilibrium. Equivalently the bulk phase can be characterised by the pressure $p$ and the bulk composition $x$ (in case of demixing in the bulk phase the transformation $\left(\mu_{1}, \mu_{2}\right) \rightarrow(p, x)$ is not unique with respect to the composition variable). The bulk fluid mixture and the adsorption problem on a single hard wall (semiinfinite system) can be studied within the same scheme [22]. From the density profiles one can define the partial mean densities $\bar{\rho}_{i}$, the total mean density $\bar{\rho}$ and the global mole fraction $\bar{x}$, as:

$$
\bar{\rho}_{i}=\frac{1}{h} \int_{0}^{h} d z \rho_{i}(z), \quad \bar{\rho}=\bar{\rho}_{1}+\bar{\rho}_{2}, \quad \bar{x}=\frac{\bar{\rho}_{1}}{\bar{\rho}} .
$$


In Sec. III we will present phase diagrams of the confined mixture in the $\mu_{1}-\mu_{2}$ plane but also in the $p-\bar{x}$ plane, since in the latter demixing regions can be appreciated. For fixed $\mu_{1}, \mu_{2}$, as $h \rightarrow \infty$ we have $\bar{x} \rightarrow x$. The equilibrium density profiles can also be obtained in other ensembles, and we have found it convenient in some cases to use the Gibbs free energy per particle $G_{N}=G / N$ which depends, in particular, on the mean pore composition $\bar{x} . G_{N}$ allows the study of fractionation effects (gaps in $\bar{x}$ ) at first-order transitions. This can be carried out by minimization of $G_{N}$ with respect to $\bar{\rho}$ and $x_{i}(z) \equiv \rho_{i}(z) / \bar{\rho}$ for a fixed value of mean pore composition $\bar{x}$. The Gibbs free energy per particle can be obtained from the Helmholtz free energy per unit volume $\mathcal{F}_{V} \equiv \mathcal{F} / V$ by a Legendre transformation, $G_{N}=\left(\mathcal{F}_{V}+\Gamma\right) / \bar{\rho}$, where $\Gamma=-\frac{\partial\left(\mathcal{F}_{V} / \bar{\rho}\right)}{\partial \bar{\rho}^{-1}}=-\Omega\left[\left\{\rho_{i}^{(\mathrm{eq})}\right\}\right] / V$, i.e. minus the grand potential per unit volume evaluated at the equilibrium density profiles [26]. Thus we obtain the usual result $\Gamma=p$, the bulk pressure, when external potentials are absent. The double-tangent construction on the function $G_{N}(\bar{x})$ for a fixed value of $\Gamma$ is equivalent to the equality of chemical potentials of different species in each of the coexisting phases. These chemical potentials in turn coincide with those appearing in the definition of $\Omega[\rho]$. Changing the value of $\Gamma$, and repeating the above procedure, we can calculate the phase diagram in the coordinates $p-\bar{x}$. Note that the bulk pressure $p$ can be calculated from the values of $\left\{\mu_{i}\right\}$.

In the following we summarise the bulk behaviour and the surface behaviour of the fluid against a single wall.

\section{Bulk phase behaviour}

In [22] we analysed the bulk phases of the above model mixture. Here we briefly discuss the main results. For the nematic phase $\rho_{i}(z)=\rho_{i}=$ const., whereas in the smectic phase $\rho_{i}(z)$ are periodic functions of $z$, with period $d$. The stability of the nematic and smectic phases was obtained in a $p-x$ (pressure-composition) phase diagram [22] (details of these calculations can be found therein). Fig. 2(a) presents the bulk phase diagram in this plane; in part (b) the same diagram is shown in the $\mu_{1}-\mu_{2}$ plane. The following features are worth mentioning: (i) there are two second-order N-S transitions (dashed lines), each starting at one of the pure-fluid cases, $x=0$ or $x=1$; (ii) one of these transitions, the one coming from the $x=0$ axis, is connected to a first-order N-S transition by a tricritical point [triangle in Fig. 2(a)]; and (iii) three regions of S-S demixing, two of them ending in corresponding 
critical points (circles), appear in the phase diagram. Note that, in the regions of smectic stability, two smectic structures occur: one where the density waves of the two species are in phase (one-layer smectic), denoted by $\mathrm{S}_{1}$, and another where the densities are out of phase (two-layer smectic), $\mathrm{S}_{2}$. The latter presents a microsegregation of the two species. By changing the conditions on the mixture one can pass from one of these smectic phases to the other in a continuous fashion. In the bulk phase diagrams of Figs. 2(a) and (b), and in the rest of the article, the smectic phases are denoted by primed or unprimed labels according to whether the smectic is rich or poor in the short component, respectively.

We now briefly comment on the expected changes in phase diagram topology if particles axes were allowed to rotate. The most important difference will be the presence of the isotropic phase, and the existence of an associated first-order isotropic-nematic phase transition with a transition gap that depends on the mixture composition. Obviously our model, with the perfect-alignment constraint, cannot account for this transition. Also, our model predicts a second-order nematic-smectic transition which, as show below, is suppressed by confinement (an effect ultimately arising from the perfect-alignment approximation). However, in situations where the bulk phases (nematic or smectic) are highly oriented, we expect a phase behavior very similar to that shown in the present work.

\section{Surface behaviour}

The behaviour of the single-wall system is very important to understand the properties of the confined mixture, as will be seen in the following section. The surface behaviour of the fluid when a nematic phase is adsorbed on a substrate was investigated in our previous work [22]. Particle axes were taken to point along the normal to the substrate, which was represented by an external potential $V_{\text {ext }}(\boldsymbol{r})$ of the same type as in (8) . We only studied the case where the bulk fluid in equilibrium with the surface structure was nematic. The nematic phase far from the wall $(z \rightarrow \infty)$ was characterised by values of the pressure $p$ and the composition $x$ or, equivalently, by the chemical potentials of the two species, $\mu_{1}$ and $\mu_{2}$. A conjugate-gradient scheme was used in the numerical minimisation (see details in Ref. [22]), from which equilibrium density distributions and values of surface tensions were obtained.

At a given value of pressure $p$, wetting of the wall by the smectic phase was found for 

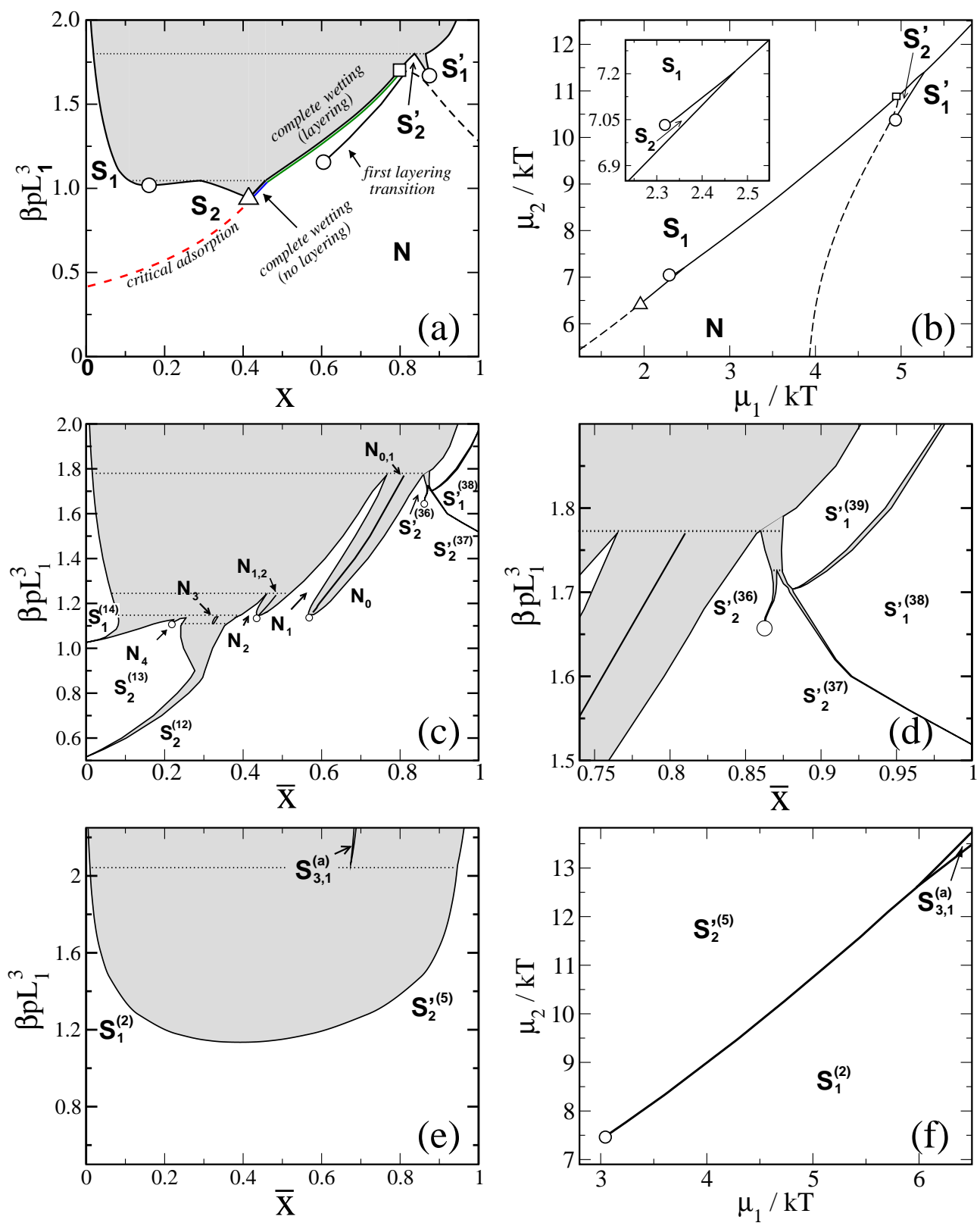

FIG. 2: (a) Bulk phase diagram of the mixture in the $p-x$ plane. Text indicates type of wetting behaviour. (b) Bulk phase diagram of the mixture in the $\mu_{1}-\mu_{2}$ plane. (c) Phase diagram of the confined mixture in the $p-\bar{x}$ plane for pore width $h=48.2 L_{1}$. (d) Zoom of the high $p$ and $\bar{x}$ region in panel (c). (e) Phase diagram of the confined mixture in the $p-\bar{x}$ plane for pore width $h=6.8 L_{1}$. (f) Phase diagram of the confined mixture in the $\mu_{1}-\mu_{2}$ plane for pore width $h=6.8 L_{1}$. In all diagrams circles indicate critical points, triangles tricritical points, squares critical end points; dashed curves are continuous phase transitions. Dotted horizontal lines indicate triple or quadruple points. 
all pressures as $x \rightarrow x_{\mathrm{NS}}(p)$, where $x_{\mathrm{NS}}(p)$ is the boundary of the N-S transition. However, depending on the bulk pressure, three régimes may occur: (i) Complete wetting by the $\mathrm{S}_{1}$ phase, rich in long particles, via an infinite sequence of surface-induced layering (SIL) transitions when $p$ is above the $\mathrm{S}_{1}-\mathrm{S}_{2}-\mathrm{N}$ triple point; at a first-order SIL transition, the composition of a localised interfacial region, with a thickness of one layer, changes abruptly from a low to a high value, as a result of which the short component is expelled from the wall and sharply localised, smectic-like layers, adsorb at the wall in a stepwise fashion, until a macroscopically thick smectic $S_{1}$ film develops at the wall (complete wetting). (ii) Complete wetting by the $\mathrm{S}_{2}$ phase without SIL transitions when $p$ is below the triple point but above the tricritical point. And (iii) critical adsorption at the second-order bulk N-S transition, i.e. for pressures below the tricritical point. The first of the infinite sequence of SIL transitions in the first régime has been plotted in Fig. 2(a); the rest are too close to the coexistence boundary to be seen. A blow up of the first four transitions is shown in Fig. 3 in two different planes. Here, and in the rest of the paper, phases denoted by $\mathrm{N}_{n}$ refer to structures with $n$ localised layers of the long particles near the wall, and a density that tends to a constant value far from the wall; in the confined case the density may not be completely uniform in the middle of the pore, but the structures are generally connected with the bulk nematic as $h \rightarrow \infty$.

\section{CONFINEMENT}

One of the most striking phenomena presented by the mixture of cylinders is the occurrence of SIL transitions at a single wall as the bulk N-S coexistence is approached. Our explanation for this phenomenon [22] is that there is an entropic competition (reduction of excluded volume) between the two species to cover the wall, which couples to translational entropy to produce a first-order phase transition. But there is another type of layering phenomena, i.e. the confinement-induced layering (CIL) transitions, which take place when a spatially ordered phase competes with a confining length scale. These phenomena are well documented and have been investigated theoretically in liquid crystals and related materials [19, 20]. CIL phenomena should also happen in fluid mixtures that can stabilise a smectic phase in bulk. Since the smectic phase of our mixture comes in two varieties $\left(\mathrm{S}_{1}\right.$ and $\left.\mathrm{S}_{2}\right)$ and the bulk phase diagram is already quite complex, we can anticipate a fascinatingly rich 

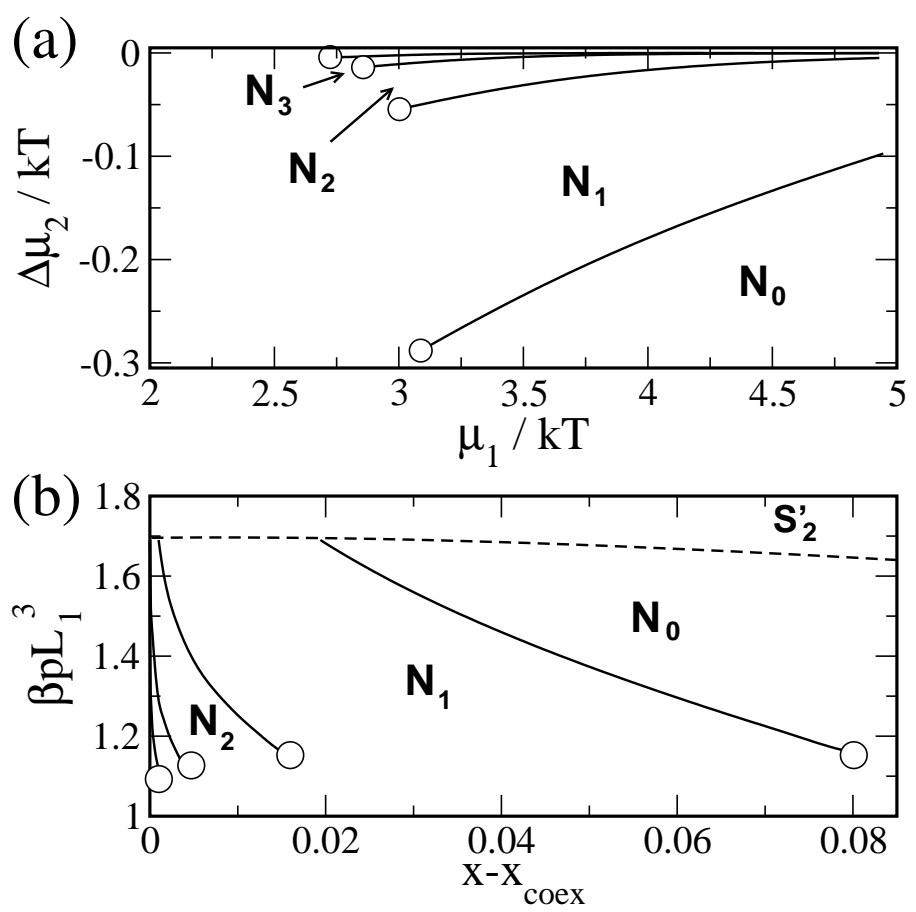

FIG. 3: SIL transitions in the planes (a) $\mu_{2}-\mu_{1}$, and (b) $p-x$ in the single-wall system. In (a), $\Delta \mu_{2}$ is the chemical potential of species 2 with respect to the value of the bulk nematic-smectic transition. In (b), $x_{\text {coex }}$ is the nematic value at the transition for each value of pressure.

phase diagram when the mixture is confined and the possible occurrence of new phases.

As explained above, the particle model used in our calculations exhibits a second-order bulk N-S transition. Due to suppression of fluctuations along the $z$ direction when the fluid is confined, the N-S transition disappears in the confined system. Therefore, it is not possible to assign in a clear manner the roles of nematic and smectic to any of the confined phases, except in some particular cases (generally speaking, as pressure or chemical potential is increased, the oscillating distribution of particles tends to become sharper throughout the pore). The particular cases are: (i) Confined phases close to $x \gtrsim 0$ and $x \lesssim 1$ existing in a closed region of stability, which are generally connected with the bulk smectic phase as $h \rightarrow \infty$. (ii) Surface phases in the confined case, which undergo SIL transitions, and which are connected with $\mathrm{N}_{n}$-type phases in the single-wall $(h=\infty)$ case (in bulk, these phases are in contact with a bulk nematic phase, but in the confined case the latter may have more or less spatial order depending on the values of pore width and pressure). Our convention is to denote all confined phases by $\mathrm{S}^{(m)}$, with $m$ the number of layers of either species inside the pore, in case (i), and by $\mathrm{N}_{n}$, with $n$ the number of absorbed layers of the long particles, 

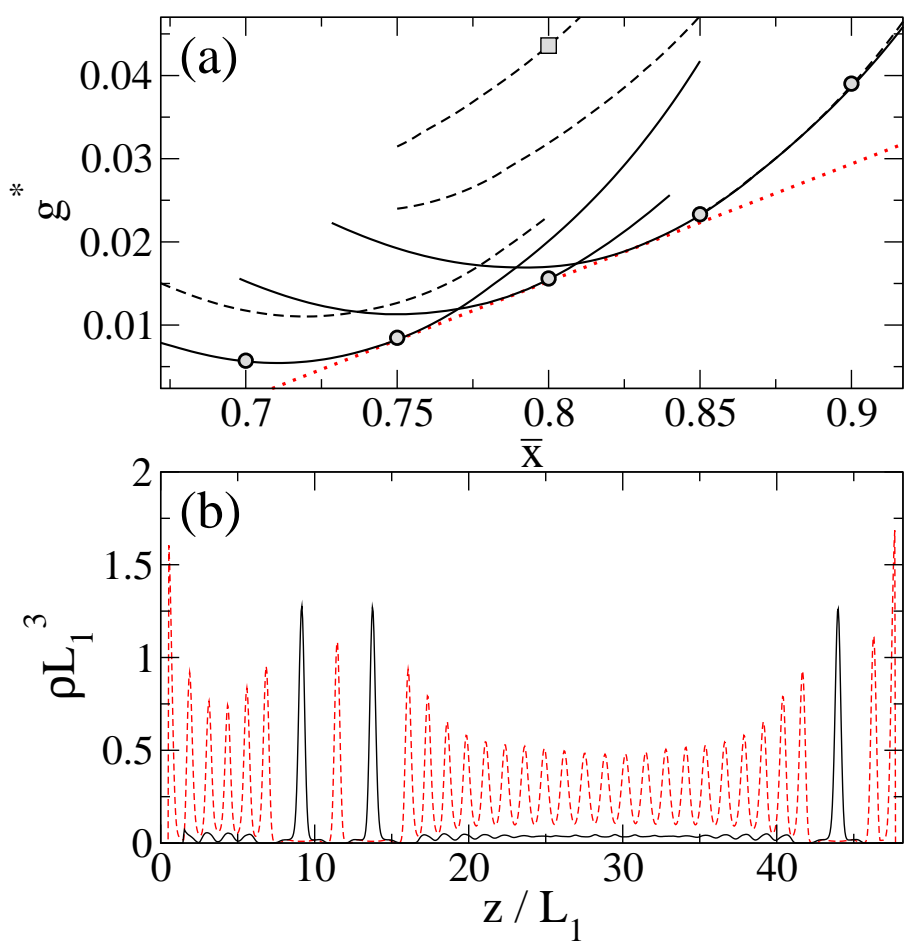

FIG. 4: (a) Gibbs free-energy density $g^{*}=G_{N} / k T$ as a function of global mole fraction $\bar{x}$ for the case $\beta p L_{1}^{3}=1.7$. For the sake of visualisation, a straight line $-10.96+6.00 \bar{x}$ has been subtracted from the free energies. Curves correspond to minima at fixed $\bar{x}$ obtained from the conjugate-gradient method (depending on the initial conditions different branches can be obtained). Continuous curves: branches that eventually lead to stable states. Dashed curves: metastable branches. Circles correspond to states obtained by simulated annealing. Dotted (straight) line indicates three-phase coexistence of a confined SIL transition. (b) Profiles of the metastable state indicated by the square in panel (a); the continuous curve is the profile of the long particles, whereas the dashed curve corresponds to the short particles.

in case (ii). Intermediate cases are not well defined by these notations.

In the confined case the method of solution of our theory is as follows. For fixed values of $\mu_{1}, \mu_{2}$, the fluid equilibrium structure within the pore was obtained using a conjugategradient (CG) method, where the $z$ coordinate is discretised as $z_{k}=k \Delta z$, with $\Delta z=0.01 L_{1}$, and $\rho_{k}^{(i)} \equiv \rho_{i}\left(z_{k}\right)$ are used as discrete variables. However, the free-energy landscape of the confined mixture is very complex, with many local minima of similar free energy, corresponding to different arrangements of particles that can accommodate into the pore. The grandpotential minimum given by the CG method depends crucially on the initial configuration 


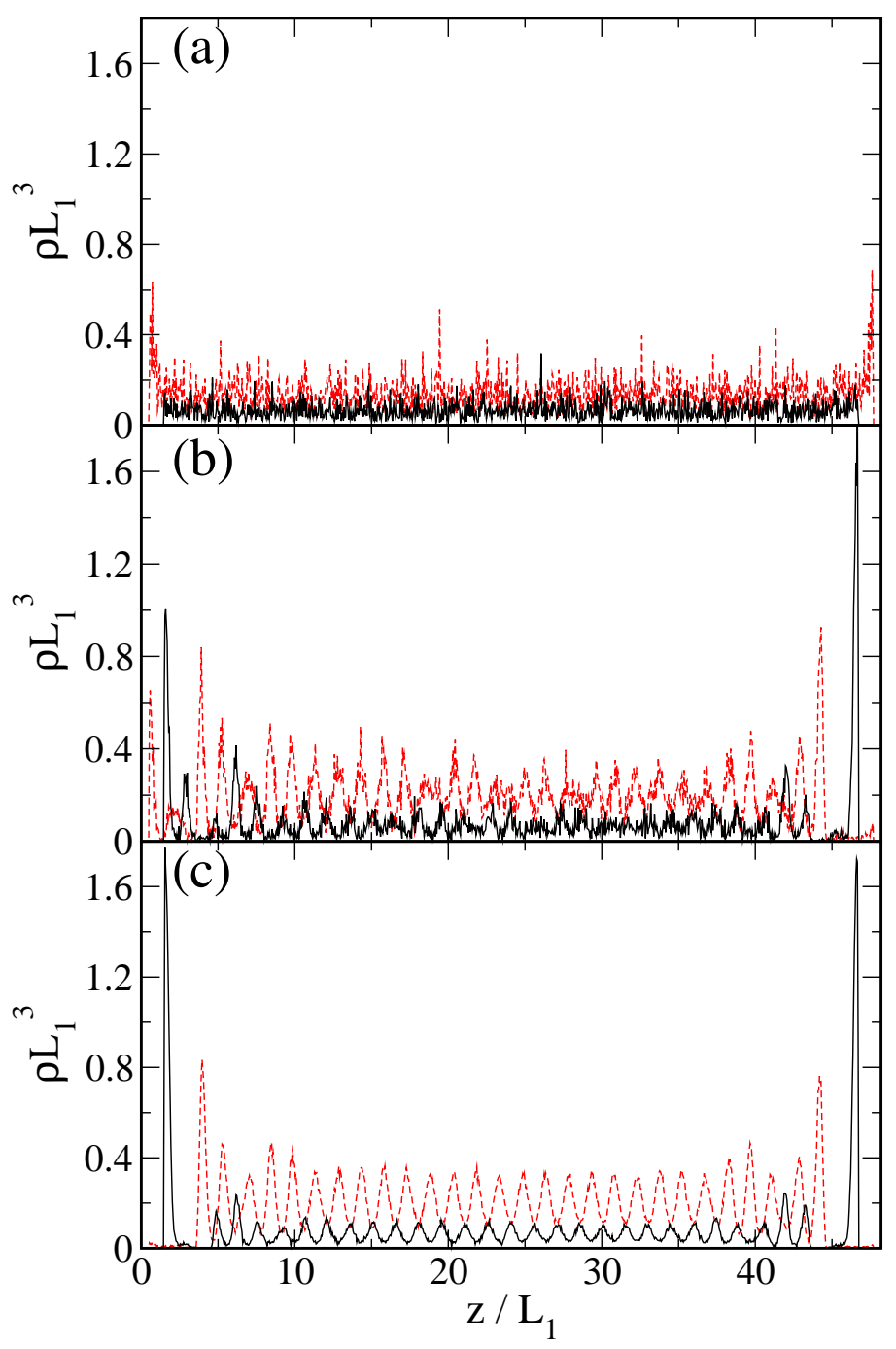

FIG. 5: From (a) to (c), sequence of profiles in a simulated-annealing simulation for the case $\beta p L_{1}^{3}=$ 1.7, $\bar{x}=0.7$ and $h=48.2 L_{1}$. The continuous curves are the profiles of the long particles, whereas the dashed curves correspond to the short particles. A complete animation of the simulation can be found in [27].

used to start the iterative CG algorithm. Alternatively, we have used a simulated-annealing (SA) method to double-check that the minima found are actually the absolute minima. Even then, one always has to bear in mind that one cannot be completely certain as to the absolute nature of the minima found, especially in particular areas of the phase diagram where the particle arrangements may be more degenerate. This is the case in the region of the phase diagram where the values of $p$ and $\bar{x}$ are high, i.e. in a compressed mixture where most of the particles are short. Fig. 4(a) shows the structure of minima in the Gibbs free-energy 
landscape as a function of $\bar{x}$ for the case $\beta p L_{1}^{3}=1.7$. Curves are minima obtained using the CG method, starting from different initial profiles. Depending on the initial condition, the minimisation procedure gets trapped into one of many possible metastable branches. Panel (b) of the figure shows a typical metastable state [actually the one located by the square in panel (a)]. For a given value of $\bar{x}$, the different metastable branches correspond to the very many different ways of distributing the layers of the long species. Circles correspond to SA simulations starting from uniform profiles which hopefully, and in the cases explored it was always so, are able to identify the correct branch. In the case shown, there exists coexistence between three structures with different values of $\bar{x}$, which follows from the double-tangent construction, for a SIL transition. Fig. 5 shows the evolution of a SA simulation for the case $\beta p L_{1}^{3}=1.7, \bar{x}=0.7$ and $h=48.2 L_{1}$. The SA procedure is able to find the minimum free-energy configuration in a few hundred steps [27]. The resulting structure is then refined using the CG algorithm. Although the difference in free energy between the coexisting phases may be relatively small (as is the case shown in Fig. 4), the values of the composition variable $\bar{x}$ are not so similar (see values at coexistence in the figure). This in turn means that the coexisting phases have different interfacial structures. Therefore, in case this scenario were to occur in an experimental system, large energy barriers separating these states may be expected, so that only fluctuations with relatively large amplitudes can take the system out from its original state. This scenario may be typical when large and moderate demixing gaps separate two stable confined phases. However, as the pore compositions of two coexisting phases become similar, and consequently also their interfacial structures become similar, these fluctuations, present in the real experimental system but not taken into account in the present model, can suppress these transitions. Inclusion of fluctuations in the orientations of the particle axes might stabilize interfacial structures such as those shown in Fig. 4 (b), which is due to the lowering of the elastic energy when particles rotate in such a way as to modify the period of the confined smectic and better commensurate the number of smectic layers with the pore width.

In order to understand the results for the confined mixture, let us first discuss the effects of confinement on the one-component fluid. The bulk fluid possesses a second-order transition from the nematic to the smectic, which disappears in the confined system due to the finite size available for spatial correlations. The only feature of the phase diagram that remains on confinement is the presence of first-order CIL transitions. These transitions occur at high 
values of pressure or chemical potential, i.e. when the smectic order inside the pore is well developed. As mentioned already, their origin is the fact that, for a given value of pore width $h$, the confined fluid can only accommodate a particular number $n$ of smectic layers, which is given approximately by $h \simeq n d$, where $d$ is the equilibrium layer spacing of the bulk smectic. In general the confined smectic will be stressed, either because it is compressed or swollen. As $h$ is varied, the number of layers will be increased or decreased to optimise the available space from a thermodynamic point of view. Layering transitions are mostly associated with variations in $h$, and depend more weakly on pressure or chemical potential, i.e. they are almost vertical in a $p$ - $h$ or $\mu$ - $h$ phase diagram. CIL transitions terminate at critical points (see Ref. [19]). There is a terminal, lower pore width at which layering transitions cease to exist.

\section{A. Wide slit pores}

Starting from the one-component fluid, as one adds a small amount of particles of the other component, the structure of CIL transitions is shifted in the phase diagram. If short particles are added to a smectic structure made of long particles, the smectic spacing will tend to increase and we expect the CIL transitions to occur at larger values of $h$. The distance between two consecutive transitions will be more or less constant. An example of this phenomenon is presented in Fig. 6(a), which shows a CIL transition between smectic phases $\mathrm{S}_{2}^{(12)}$ and $\mathrm{S}_{1}^{(13)}$, containing 12 and 13 layers, respectively, in a mixture at low pressure $\left(\beta p L_{1}^{3}=0.81\right)$. Note that there is a mole-fraction gap at the transition, i.e. the global mole fractions $\bar{x}$ of the two coexisting structures are different. There is also a terminal pore width beyond which the layering transition disappears. Above the transition gap in Fig. 6(a) the

coexisting phase $\mathrm{S}_{1}^{(13)}$ smoothly changes to an out-of-phase structure $\mathrm{S}_{2}^{(13)}$. On the other side of the phase diagram $(x \lesssim 1)$, CIL transitions are not occurring at this value of the pressure since the mixture is in the $\mathrm{N}$ bulk regime, but they would certainly occur at higher pressure.

At higher pressures the phenomenology becomes much more complex, as CIL transitions compete with demixing. This was to be expected, as the bulk phase diagram shows that demixing occurs for pressures $\beta p L_{1}^{3} \gtrsim 1.0$. An example is shown in Fig. 6(b). For the pressure shown, $\beta p L_{1}^{3}=1.51$, strong N-S demixing occurs at bulk [Fig. 2(a)], and 

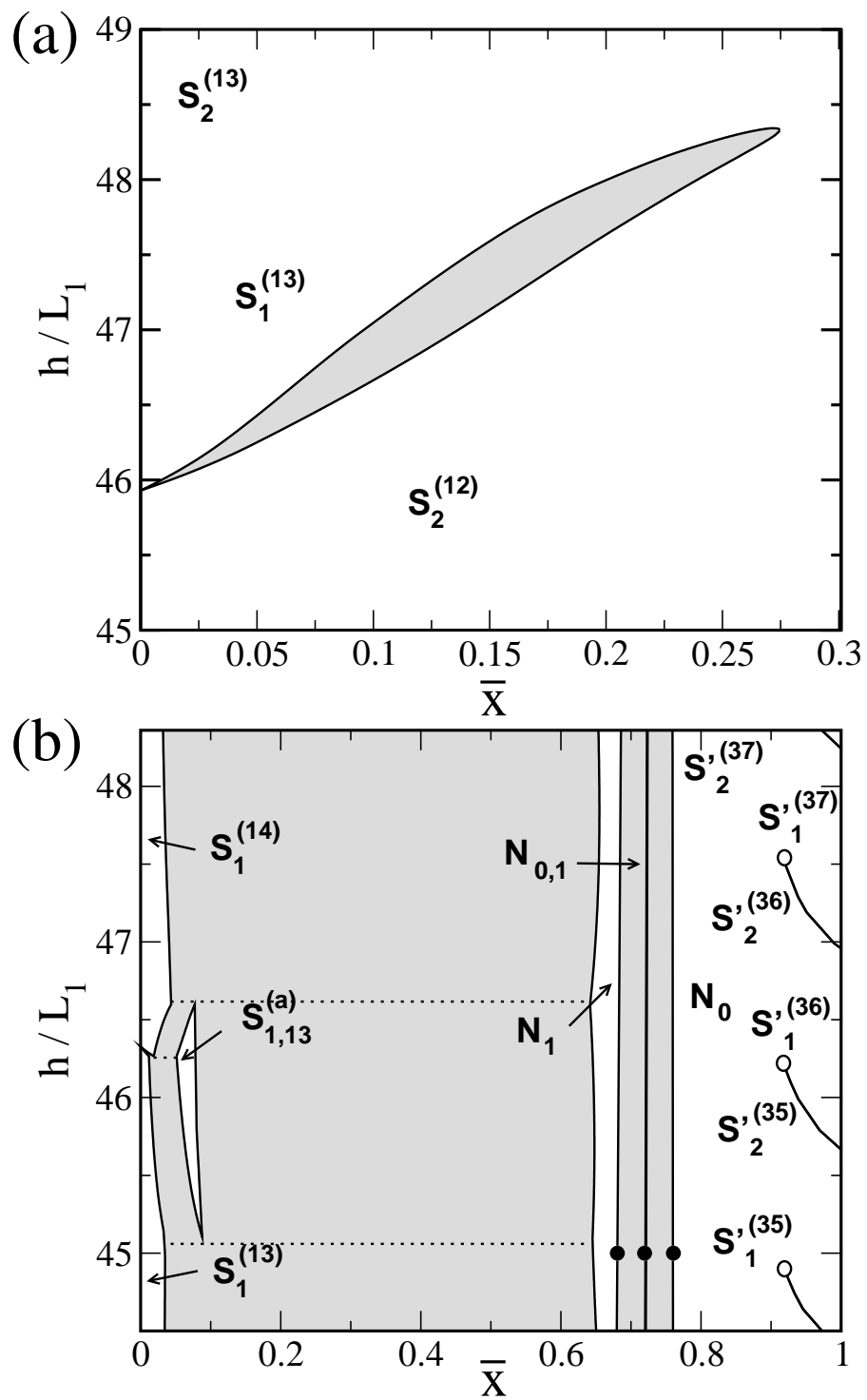

FIG. 6: Phase diagrams of the confined mixture in the $h-x$ plane for two values of the pressure. (a) $\beta p L_{1}^{3}=0.81$. (b) $\beta p L_{1}^{3}=1.51$. Labels indicate nature of phases. Horizontal dotted lines denote triple points.

correspondingly the confined mixture also exhibits demixing in a similar mole-fraction interval. But with important differences, which will be commented on later. First note that, in the range $x \lesssim 1$, smectic CIL transitions of the same type as in the region $x \gtrsim 0$ for the lower pressure do exist. They are separated approximately by a pore-width increment $\Delta h \simeq 1.2 L_{1} \simeq d$, the bulk smectic spacing of the short species. Fig. 7 shows the density profiles of two phases that coexist at the $\mathrm{S}_{2}^{\prime(35)}-\mathrm{S}_{1}^{\prime(36)}$ CIL transition.

In the region $\bar{x} \gtrsim 0$, CIL transitions are rapidly preempted by a demixing instability. One 


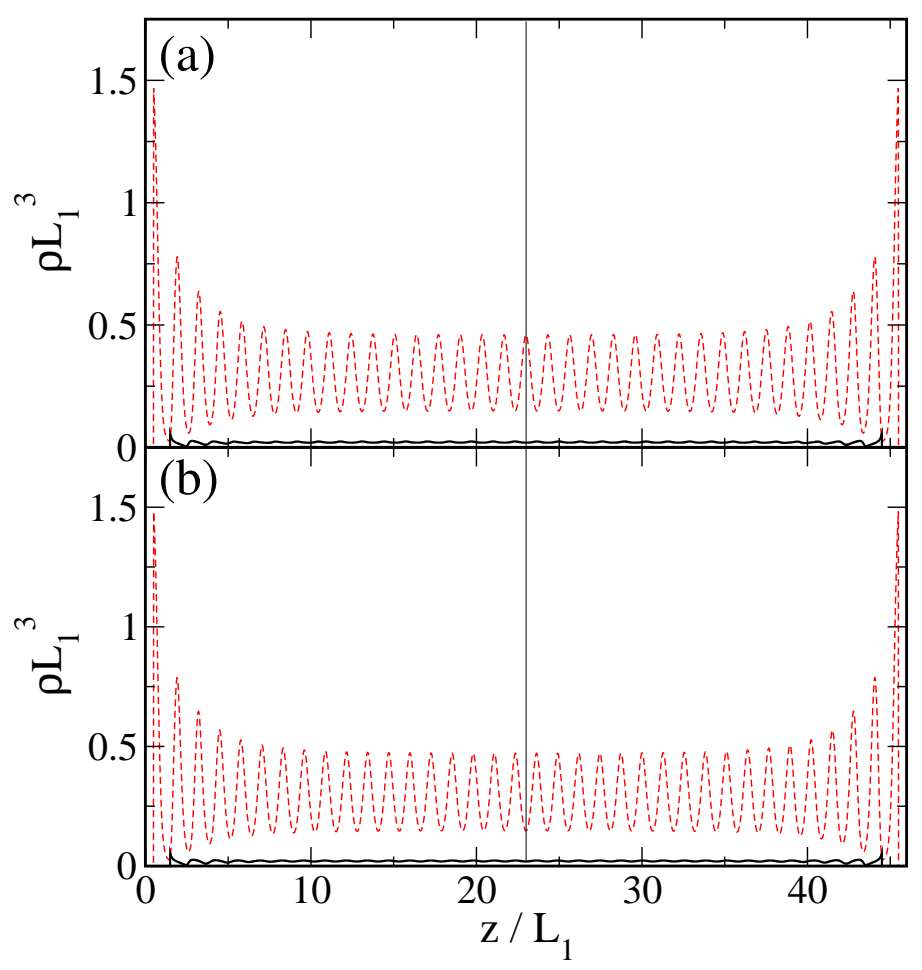

FIG. 7: Density profiles of two phases that coexist at a CIL transition in the confined fluid with $\beta p L_{1}^{3}=1.51$ and $h=46.0 L_{1}$ [see Fig. 6(b)]. (a) $\mathrm{S}_{2}^{\prime(35)}$ phase, with $\bar{x}=0.9385$. (b) $\mathrm{S}_{1}^{\prime(36)}$ phase, with $\bar{x}=0.9391$. The thin vertical line divides the slit pore in two identical parts. Continuous curve: species 2 (long particles). Dashed curve: species 1 (short particles).

interesting feature of the phase diagram of Fig. G(b) is that, in the proximity of the CIL transition, an asymmetric structure, denoted by $S_{1,13}^{(a)}$, is stabilised in a small island in the $h-\bar{x}$ plane; presumably, these islands exist associated with other CIL transitions, creating phases $\mathrm{S}_{n, m}^{(\mathrm{a})}$ (in this notation, $n$ and $m$ are the number of layers of species 1 and 2 of the asymmetric structure, respectively). In the case shown, the island interacts with the CIL and demixing transitions, creating triple points where three confined fluids coexist. For the $\mathrm{S}_{1}^{(13)}-\mathrm{S}_{1}^{(14)}$ CIL transition, the asymmetric structure, $\mathrm{S}_{1,13}^{(\mathrm{a})}$, contains 7 smectic layers of long particles at one wall, separated from 6 smectic layers of long particles at the other wall, by a single localised layer of the (minority) short species. Note that other very similar structures with almost identical values of free energy but with different positions of the single peak of the short particles may exist, but structures with more than one layer of short particles, for larger values of $\bar{x}$, are unstable with respect to demixing. The triple points are indicated by horizontal dotted lines in Fig. 6(b), while the density profiles of the three phases that 


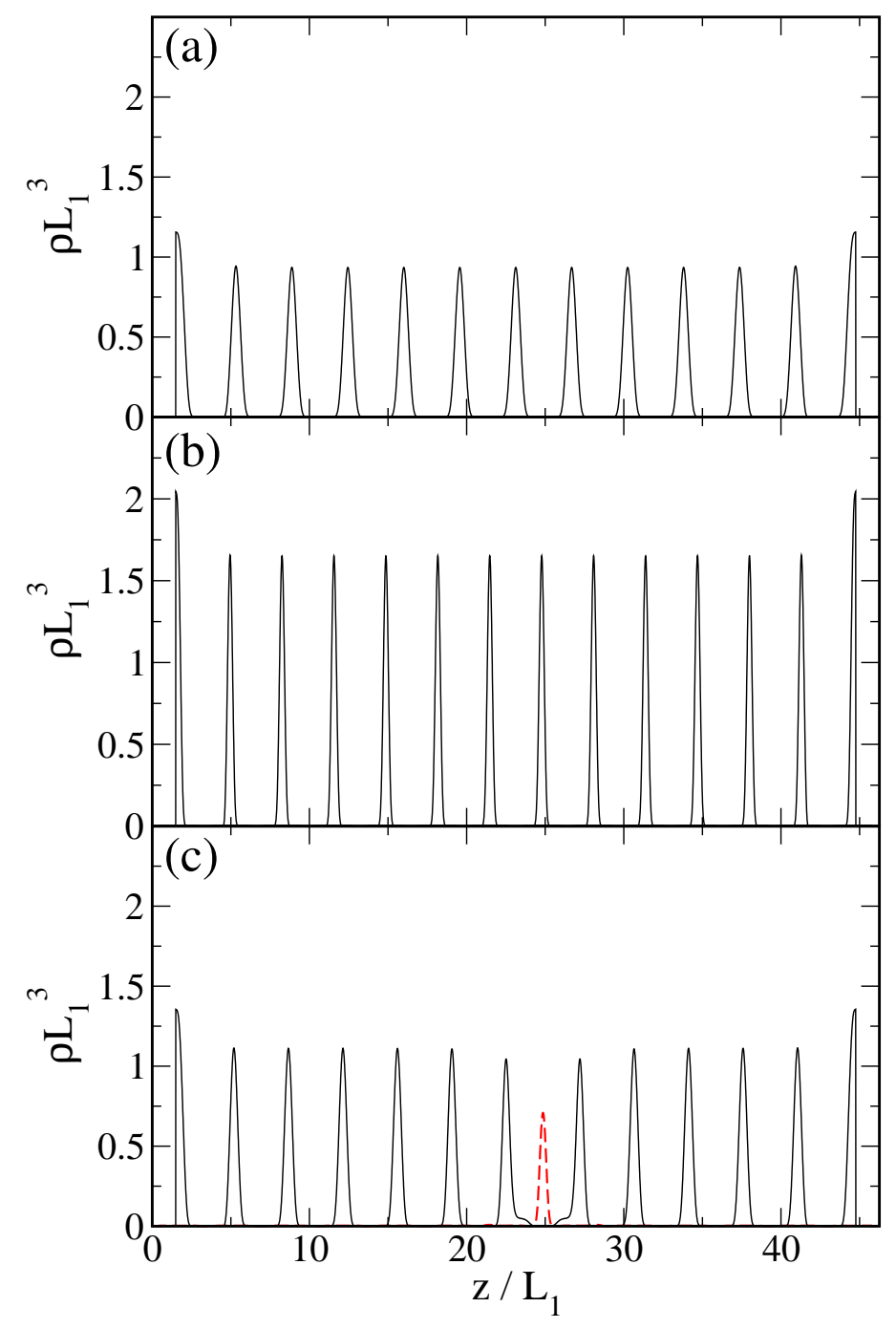

FIG. 8: Density profiles of the three phases that coexist at a value of pressure $\beta p L_{1}^{3}=1.51$ and for a pore width $h=46.3 L_{1}$ [see Fig. 6(b)]. (a) $S_{1}^{(13)}$ phase, with $\bar{x}=0.012$. (b) $S_{1}^{(14)}$ phase, with $\bar{x}=0.019$. (c) $\mathrm{S}_{1,13}^{(\mathrm{a})}$, with $\bar{x}=0.053$. Continuous curve: species 2 (long particles). Dashed curve: species 1 (short particles). Note that, in panels (a) and (b), the density of short particles is too small to be visible in the graphs.

coexist at the intermediate triple point, involving the $S_{1}^{(13)}, S_{1}^{(14)}$ and $S_{1,13}^{(a)}$ phases, can be seen in Fig. 8

Leaving aside these islands of stability within the demixing region, we can see that this region consists of two segregation 'bands': a first, wide band, in the mole-fraction interval $0.04 \lesssim \bar{x} \lesssim 0.65$, and a second, narrow band, in the interval $0.68 \lesssim \bar{x} \lesssim 0.76$. The first band corresponds to the shifted bulk demixing N-S transition, while the second is associated 


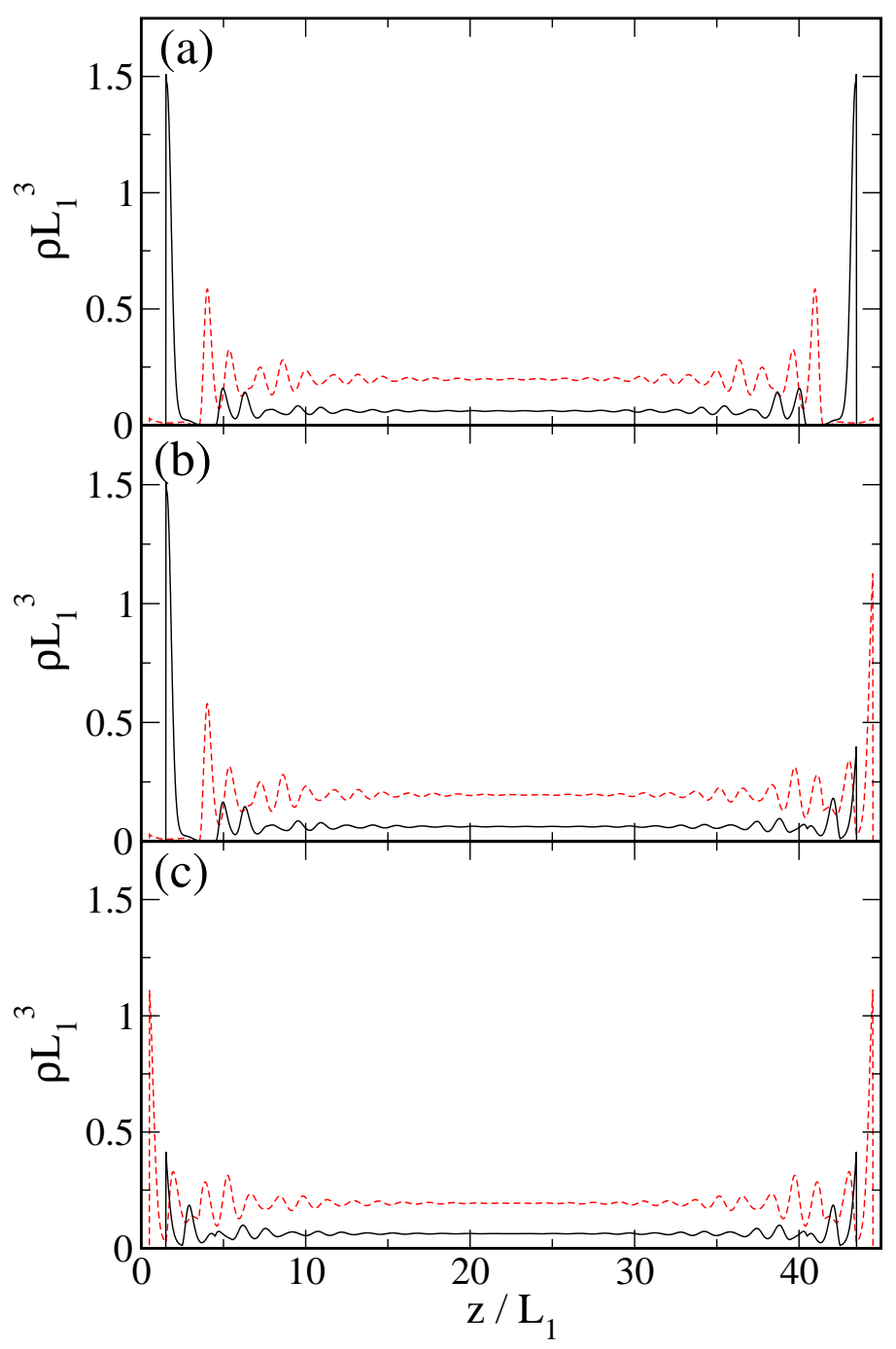

FIG. 9: Density profiles of the three phases that coexist at a value of pressure $\beta p L_{1}^{3}=1.51$ and for a pore width $h=45.0 L_{1}$ [see Fig. 6(b)]. (a) $\mathrm{N}_{1}$ phase, with $\bar{x}=0.688$. (b) $\mathrm{N}_{0,1}$ phase, with $\bar{x}=0.724$. (c) $\mathrm{N}_{0}$ phase, with $\bar{x}=0.759$. Continuous curve: species 2 (long particles). Dashed curve: species 1 (short particles).

with the SIL transitions occurring in the single-wall system, but here in the confined case. Inspection of Fig. 9 indicates that this is indeed the case. In the figure, density profiles that coexist at a pressure $\beta p L_{1}^{3}=1.51$ in a pore of width $h=46.3 L_{1}$ are shown [filled circles in Fig. [6(b)]. Profiles in panels (a) and (c) are symmetric and correspond to the phases with low and high values of mole fraction, respectively. The structural differences between the two profiles are identical to those occurring at a single-wall interfacial structure undergoing a SIL transition, described in Sec. IID The mole-fraction gap at this SIL transition arising 
in the confined fluid tends to decrease as $h$ is decreased, until it eventually disappears at a critical point, not shown in Fig. 6(b). The transition is connected to a corresponding SIL transition at a single surface as $h \rightarrow \infty$. Inside the instability region of the confined SIL transition an almost vertical, continuous line has been drawn, which corresponds to a peculiar structure that coexists with the other two [panel (b) in Fig. 9]. This structure, which has interfacial structures identical to that of (a) at the left wall and to that of (b) at the right wall, is asymmetric but has the same interfacial free energy as the other two (an equivalent structure is obtained by interchanging the right and left interfacial profiles). The existence of structure (c) is the consequence of this phenomenon being associated to the SIL transitions, which in the confined case, and for wide pores, occur at both surfaces independently without any interaction. As the pore width is reduced, interaction between the two surfaces structures would make the line inside the instability region in Fig. 6(b) to have a finite width. This structure seems too elusive to be detected experimentally, since domains of the two coexisting interfacial structures would probably mix in different proportions at both surfaces.

The $p-\bar{x}$ phase diagram of the confined mixture for a particular value of pore width, $h=48.2 L_{1}$ (in the regime of wide pores) is plotted in Fig. 2(c). The diagram contains the same features as previously described, but viewed in a different way. Also, it allows to visualise critical points associated with confined SIL transitions and some additional features. The diagram is to be compared with the corresponding bulk diagram. The wide demixing region is quite similar. But some differences are apparent. First, as mentioned before, the second-order bulk N-S transition is vanished. Second, the structure of CIL transitions appears in the regions $x \gtrsim 0$ and $x \lesssim 1$, and is seen to either interact with demixing or terminate in critical points. Since $h$ is fixed, the number of transitions in the phase diagram is very limited (CIL transitions occur mainly with respect to pore width and are weakly dependent on pressure). In Fig. 10 the density profiles of three representative structures associated with the CIL transitions are shown. These phases are labelled as $\mathrm{S}_{2}^{(12)}$, $\mathrm{S}_{2}^{(13)}$ and $\mathrm{S}_{1}^{(14)}$. In the first two, the density profiles of the two species are out of phase, whereas in the third the minority (short) species adopts an in-phase configuration with two small, separated peaks about the main smectic layer; in this case the short particles go into the main layer, forming a wide two-in-one layer where short particles flow more or less freely.

An important feature in the phase diagram of Fig. 2(c) is that the remnant of some of 


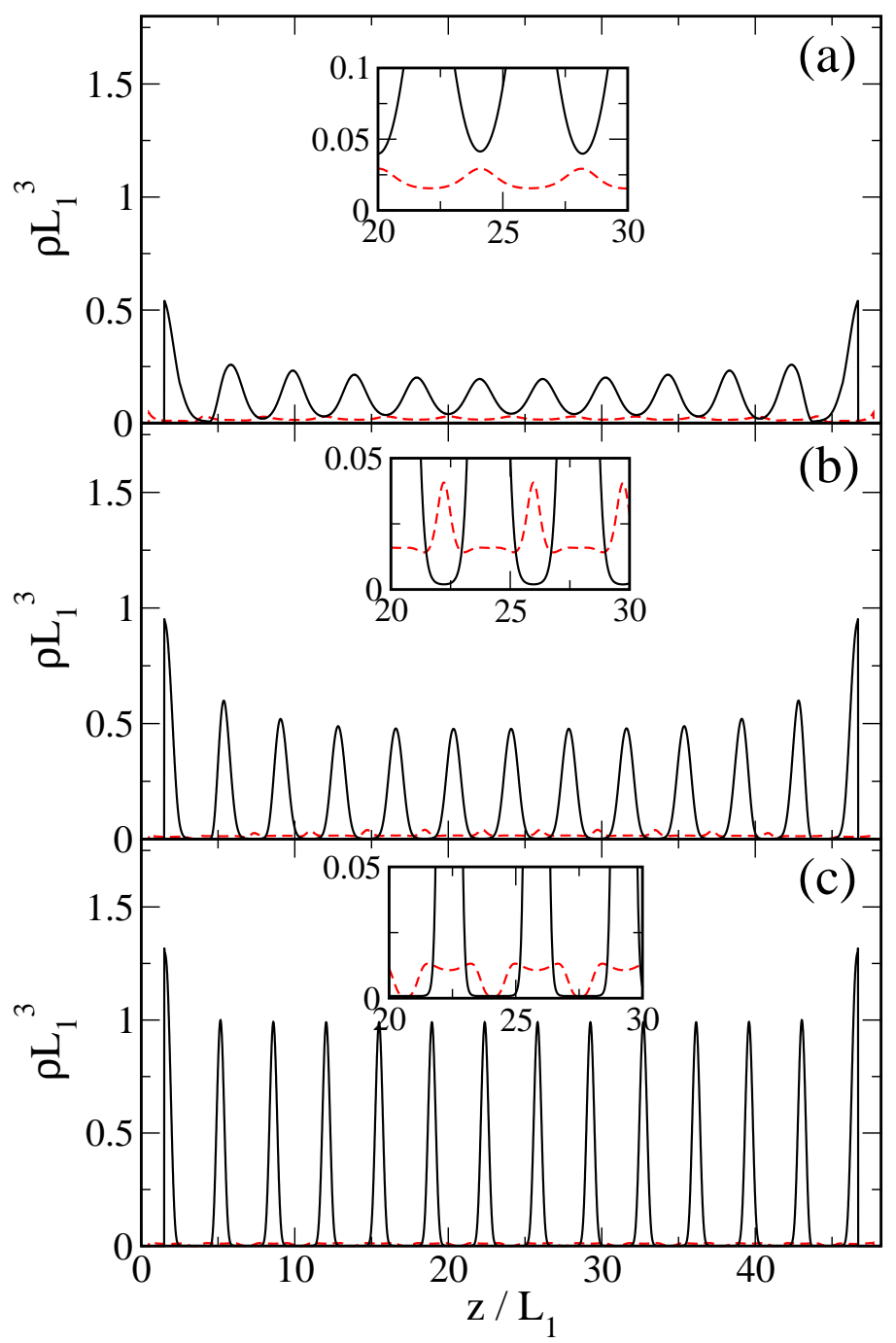

FIG. 10: Density profiles of three representative states labelled in Fig. $2\left(\right.$ c) as $\mathrm{S}_{2}^{(12)}, \mathrm{S}_{2}^{(13)}$ and $\mathrm{S}_{1}^{(14)}$ for a pore width $h=48.2 L_{1}$. (a) $\mathrm{S}_{2}^{(12)}$ phase: $\beta p L_{1}^{3}=0.6, \bar{x}=0.14$; (b) $\mathrm{S}_{2}^{(13)}$ phase: $\beta p L_{1}^{3}=1.0$, $\bar{x}=0.10 ;$ and $(\mathrm{c}) \mathrm{S}_{1}^{(14)}$ phase: $\beta p L_{1}^{3}=1.2, \bar{x}=0.05$. Continuous curves: species 2 . Dashed lines: species 1.

the (infinitely many) SIL transitions occurring in the single-wall case are clearly apparent. For the pore width shown, $h=48.2 L_{1}$, only four of them survive. The first one, involving the phases $\mathrm{N}_{0}-\mathrm{N}_{1}$, exhibits a long segregation region ending in a critical point; this structure is reminiscent of the corresponding $\mathrm{N}_{0}-\mathrm{N}_{1}$ transition in the single-wall system, and in the variable $\bar{x}$ the transition shows a gap. The second transition, $\mathrm{N}_{1}-\mathrm{N}_{2}$, is much shorter but exhibits the same characteristics. The third transition occurs right in the middle of the demixing region and, instead of being a separated segregation region, it appears as a very 


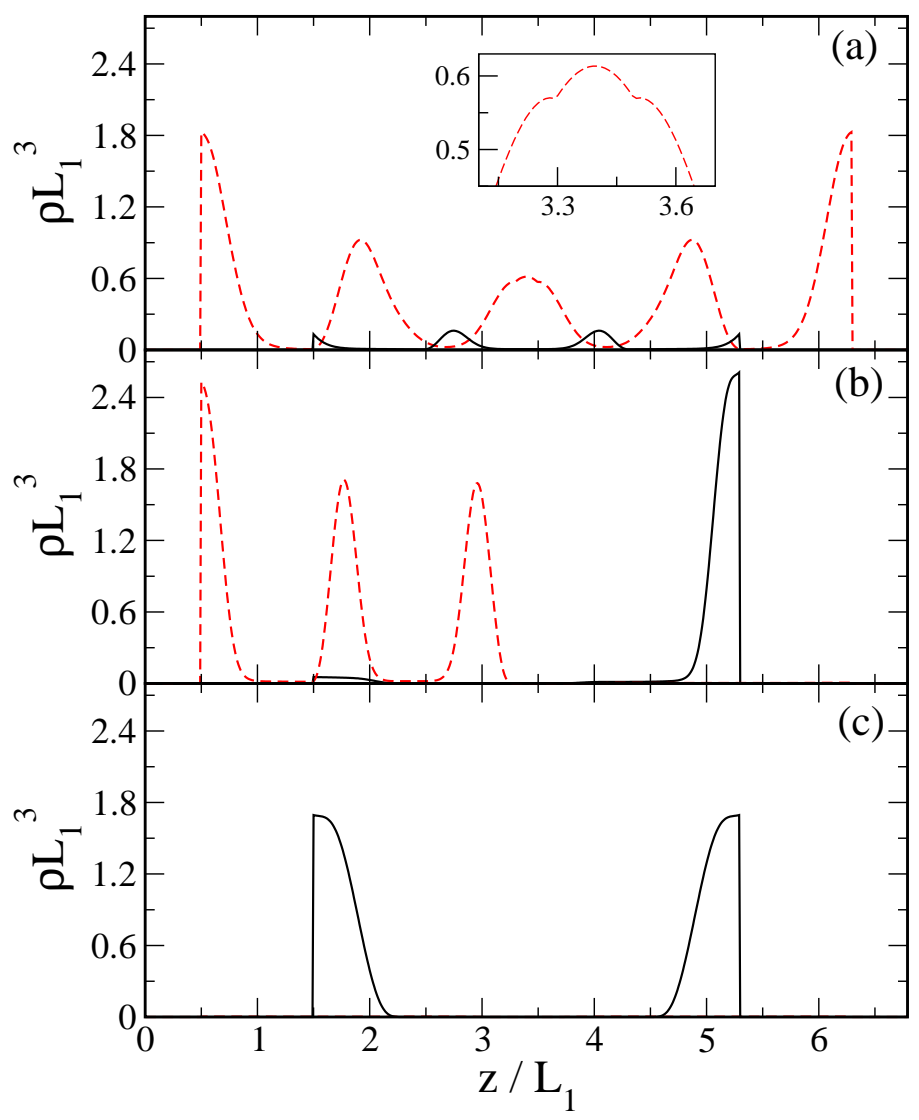

FIG. 11: Density profiles of three states that coexist at $\beta p L_{1}^{3}=2.0$ with pore width $h=6.8 L_{1}$ [see Fig. 2(e)]. (a) $\mathrm{S}_{2}^{\prime(5)}$ phase, with $\bar{x}=0.944$; (b) $\mathrm{S}_{3,1}^{(a)}$ phase, with $\bar{x}=0.674$, and (c) $\mathrm{S}_{1}^{(2)}$ phase, with $\bar{x}=0.010$. Continuous curves: species 2. Dashed curves: species 1 .

small stability island $\left[\mathrm{N}_{3}\right.$ in Fig. 2(c)]. Finally, the $\mathrm{N}_{3}-\mathrm{N}_{4}$ transition is very short, and again presents an associated critical point. As mentioned in the discussion on the $h-\bar{x}$ phase diagram, Fig. 6(b), asymmetric phases appear in the central region of the mole-fraction gap in the case of the first two SIL transitions; these structures are indicated by the labels $\mathrm{N}_{0,1}$ and $\mathrm{N}_{1,2}$ in Fig. 2(c).

Finally, we focus on the region at the upper-right corner of the phase diagram of Fig. 2(c). This region is quite complex. A zoom of this region is presented in Fig. 2(d). The small demixing region separating the bulk $\mathrm{S}_{1}^{\prime}$ and $\mathrm{S}_{2}^{\prime}$ phases, with the corresponding critical point, still survives for this pore width. Again, the bulk second-order N-S transition disappears, but the CIL transitions associated with the one-component short-particle fluid survive in mixtures with up to $12 \%$ of long particles. These transitions interact with the small demixing region. A final feature in Fig. 2(d) is the existence of a quadruple point, indicated by the 

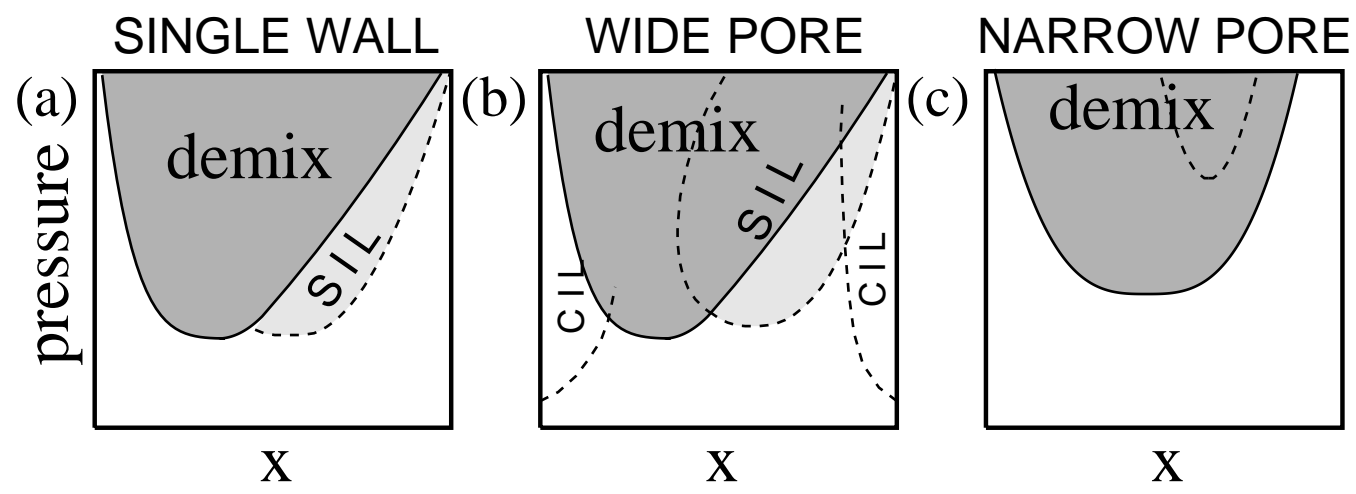

FIG. 12: Schematic of phase diagram evolution as the slit pore width is reduced.

horizontal dotted line.

\section{B. Narrow slit pores}

In the case of narrow pores, available space is much more limited and much of the rich phenomenology described for wide pore vanishes. We have investigated the case $h=6.8 L_{1}$. The $p-\bar{x}$ phase diagram is depicted in Fig. 2(e). A large demixing region persists, but the diagram is otherwise relatively featureless: both SIL and CIL transitions seem to have disappeared, and the only feature that remains is a very narrow stability region, deep in the demixing region, where a largely asymmetric structure is stabilised. As a result, a new triple point arises. This asymmetric structure might be the remnant of a SIL transition in wider pores. Fig. 11 shows the density profiles of the three phases that coexist at the triple point. The phase shown in panel (b) may be interpreted as a demixed phase where mixing entropy is compensated by an optimised surface entropy. Fig. 2(f) is the corresponding phase diagram in the $\mu_{1}-\mu_{2}$ plane. The demixing transition is slightly shifted with respect to the corresponding bulk transition [see Fig. 2(b)].

\section{Kelvin equation}

The macroscopic approach to capillary transitions is given by the Kelvin equation, which relates the degree of under- (or over-) saturation of the capillary phase transition, with respect to a reservoir, in say chemical potential, $\Delta \mu$, to the surface tension $\gamma_{\alpha \beta}$ of the 
interface between the two coexisting phases $\alpha$ and $\beta$, the contact angle $\Theta$ and the pore width $h$ (in the case of a wetting layer, the pore width has to be diminished by twice the thickness of the wetting layer $w$ ). The approach is only valid asymptotically, in the regime where $h \rightarrow \infty$. For a mixture, the Kelvin equation has been discussed by Evans and Marini Bettolo Marconi [28]. We can apply it to our particular capillary demixing system. Let $\alpha$ denote the phase favoured by the walls, in our case the $S_{1}$ phase, and $\beta$ the phase present in the central region of the pore, in our case N. We should remind ourselves that the real coexisting pore structure, $\mathrm{N}_{n}$, consists of two 'smectic' films adsorbed on the walls with $n$ layers each and a central, more or less uniform, nematic film (the value of $n$ depends on $p$ and $h$. In a very wide pore, $n$ will be large and it makes sense to split the $\mathrm{N}_{n}$ structure into two adsorbed films and a central one; in a narrower pore, the smectic layers in $\mathrm{N}_{n}$ may be just a few in number, and such a division, and as a consequence Kelvin equation, is not valid). Then the shift in chemical potential $\Delta \mu_{2}$ of the long species (more abundant in the $\alpha$ phase) with respect to the bulk value is given by [28]

$$
\Delta \mu_{2}=\frac{2 \gamma_{\alpha \beta} B^{-1} \cos \Theta}{h \rho_{1}^{(\alpha)}},
$$

where the $B$ coefficient is

$$
B=\left(\frac{1-x}{x}\right)_{\alpha}-\left(\frac{1-x}{x}\right)_{\beta}=\frac{x_{\beta}-x_{\alpha}}{x_{\alpha} x_{\beta}} .
$$

and where the composition values are given at bulk. Since we have wetting at bulk coexistence, $\cos \Theta=1$ and $h \rightarrow h-2 w$. To check the validity of the equation in our present setup, we have applied the equation for a pressure $\beta p L_{1}^{3}=1.25$ and for the wider pore width used in this work, $h L_{1}^{-1}=48.2$ (in the case of the narrower pore, $h L_{1}^{-1}=6.8$, the whole procedure is meaningless, as there is no way to identify smectic and nematic films in the fluid structure, since the pore is too narrow). We have $w \approx 2 L_{2}=6$ [for $\beta p L_{1}^{3}=1.25$ we have coexistence between the $\mathrm{S}_{1}$ and $\mathrm{N}_{2}$ phases, see Fig. 2(c)], $x_{\alpha}=0.0588, x_{\beta}=0.5823$, $\beta \gamma_{\alpha \beta} L_{1}^{2}=0.055451$, and $\rho_{1}^{(\alpha)} L_{1}^{3}=0.0103$, which gives $B^{-1}=0.0654$ and $\beta \Delta \mu_{2}=0.019$. This is to be compared with $\beta \Delta \mu_{2}=0.029$ from the density-functional calculation. The discrepancy is a huge $35 \%$, and the reason is twofold: (i) the pore width $h=48.2 L_{1}$ only spans approximately 15 lengths of the long particle; this is too small for the Kelvin equation to be accurate. (ii) In confined layered phases, such as the smectic phase, there occur important commensuration effects between the intrinsic periodicity of the phase and the pore width, 
and an elastic contribution, containing the layer compressibility modulus, to the Kelvin equation must be included [17, 19]. For wide pores the simple Kelvin equation would give predictions for the capillary phase transition that are roughly averages over the real values (which are oscillatory with respect to the pore width, with a period of approximately one particle length).

\section{CONCLUSIONS}

In this paper we have discussed the phenomenology of a confined mixture of hard cylindrical particles oriented in the direction perpendicular to the confining hard walls. Competition between the pore width $h$ and the two lengths of the particles, $L_{1}$ and $L_{2}$, create a complex behaviour. Three regimes can be identified, see Fig. 12, The first is the single-wall case, $h=\infty$ [Fig. 12(a)], where there is a wide demixing region and SIL transitions induced by the wall-fluid interaction; these off-coexistence transitions are associated with a regime of complete wetting by a smectic phase when nematic conditions prevail at bulk. In the second regime, that of wide but finite pores, $L_{1}, L_{2} \ll h<\infty$ [Fig. 12(b)], a new feature arises: CIL transitions, induced by confinement, when the composition of the mixture is close to zero or unity (i.e. almost one-component cases), which may or may not interact with a wide demixing region. SIL transitions are affected by the confinement, and only a finite number of them survive. Finally, in the regime of narrow pores, i.e. wall separations only slightly larger than both particle lengths, $h>L_{1}, L_{2}$ [Fig. 12(c)], only demixing survives, and weak signatures of the layering transitions may be seen in the form of narrow stability islands in the demixing mole-fraction gap. As expected for mixtures of particles with such different volumes, demixing is always quite strong. The segregation region shows a surprisingly invariant mole-fraction gap, and quite similar values for the pressure above which demixing occurs, with respect to pore width. This is reflected in Fig. 13, where the demixing regions for the three cases investigated in this paper have been superimposed.

This work has focused on confined phases with smectic symmetry. The possible stability of the columnar phase is a question that is left for future work. A complete calculation of the absolute stability of the columnar phase in the context of the present model is a difficult task which will have to be tackled using numerical techniques different from the

ones developed here. In our previous work [22] we used a bifurcation analysis to show that 


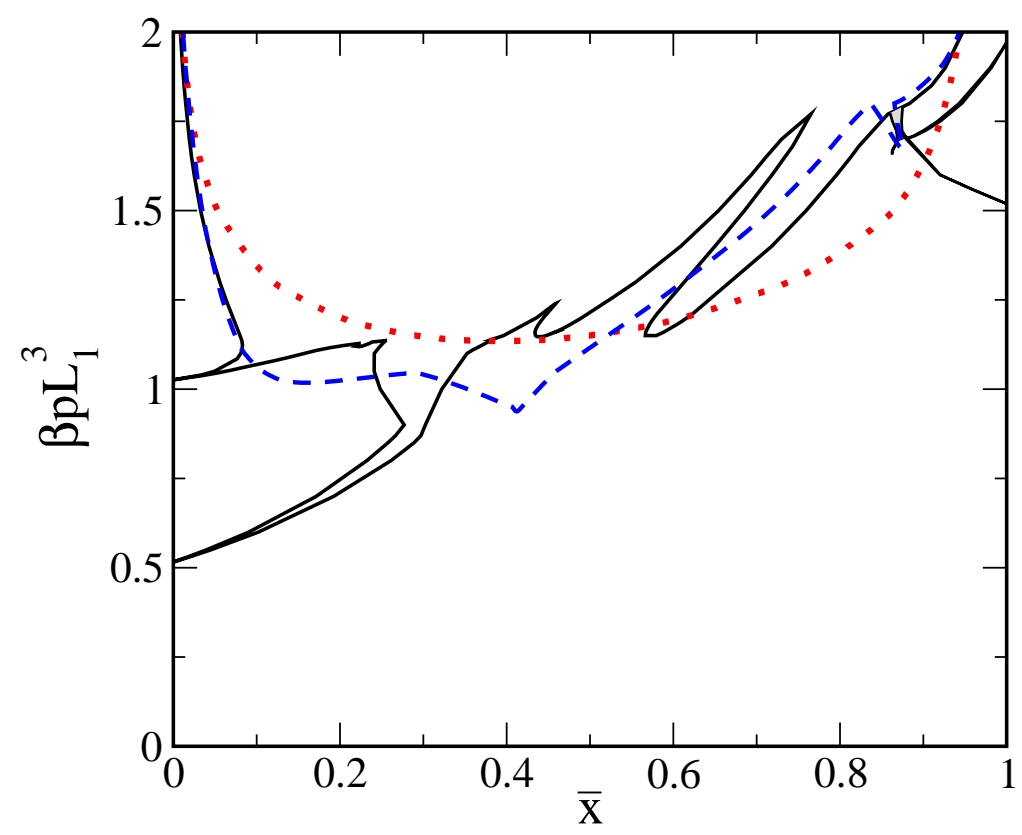

FIG. 13: Demixing regions in the plane $p-\bar{x}$ for the three cases investigated in this work: $h=\infty$ (dashed curve), $h=6.8 L_{1}$ (dotted curved) and $h=48.2 L_{1}$ (continuous curve).

the nematic-columnar spinodal line is always below the nematic-smectic line for all values of composition. This is not a proof that the smectic phases are stable with respect to the columnar phase, but at least indicates that part of the phases obtained here could be stable in some range of pressures.

\section{Acknowledgments}

We acknowledge support from the Dirección General de Universidades e Investigación of the Comunidad de Madrid (Spain), under the R\&D Programmes of activities MODELICOCM/S2009ESP-1691 and NANOFLUID, and to the Ministerio de Educación y Ciencia of Spain (grants FIS2007-65869-C03-01, FIS2008-05865-C02-02 and MOSAICO).

[1] J.-H. Ahn, H.-S. Kim, K. J. Lee, S. Jeon, S. J. Kang, Y. Sun, R. G. Nuzzo and J. A. Rogers, Science 314, 1754 (2006).

[2] Y. Niidome, Y. Nakamura, K. Honda, Y. Akiyama, K. Nishioka, H. Kawasaki and N. Nakashima, Chemical Communications, 1754 (2009). 
[3] S. Ramanathan, S. Patibandla, S. Bandyopadhyay, J.D. Edwards and J. Anderson, J. Mater. Sci.: Mater. Electron 17, 651 (2006).

[4] T. J. Morrow, M. Li, J. Kim, T. S. Mayer and C. D. Keating, Science 323, 352 (2009).

[5] L. D. Gelb, K. E. Gubbins, R. Radhakrishnan and M. Sliwinska-Bartkowiak, Rep. Prog. Phys. 62, 1573 (1999).

[6] M. Schoen, D. J. Diestler, and J. H. Cushman, J. Chem. Phys. 87, 5464 (1987).

[7] C. L. Rhykerd Jr, M. Schoen, D. J. Diestler and J. H. Cushman, Nature 330, 461 (1987).

[8] M. Schoen, J. H. Cushman, D. J. Diestler and C. L. Rhykerd, J. Chem. Phys. 88, 1394 (1988).

[9] S. Auer and D. Frenkel, Phys. Rev. Lett. 91, 015703 (2003).

[10] A. Patrykiejew, L. Salamacha and S. Sokolowski, J. Chem. Phys. 118, 1891 (2003).

[11] L. Salamacha, A. Patrykiejew, S. Sokolowski and K. Binder, J. Chem. Phys. 120, 1017 (2004).

[12] M. Dijkstra, Phys. Rev. Lett. 93, 108303 (2004).

[13] L. Salamacha, A. Patrykiejew, S. Sokolowski and K. Binder, J. Chem. Phys. 122, 074703 (2005).

[14] A. Patrykiejew and S. Sokolowski, J. Chem. Phys. 124, 194705 (2006).

[15] A. Fortini, M. Schmidt and M. Dijkstra, Phys. Rev. E 73, 051502 (2006).

[16] A. Fortini and M. Dijkstra, J. Phys.: Condens. Matter 18, L371 (2006).

[17] A. Ciach, Bulletin of the Polish Academy of Sciences (Technical Sciences) 55, 179 (2007)

[18] See e.g. Liquid crystals: applications and uses, vol. 1, Edited by B. Bahadur (World Scientific, Singapore, 1990).

[19] D. de las Heras, E. Velasco and L. Mederos, Phys. Rev. Lett. 94, 017801 (2005); D. de las Heras, E. Velasco and L. Mederos, Phys. Rev. E 74, 011709 (2006).

[20] M. Tasinkevych and A. Ciach, Phys. Rev. E 72, 061704 (2005).

[21] Y. Martínez-Ratón, Phys. Rev. E 75, 051708 (2007).

[22] D. de las Heras, Y. Martínez-Ratón and E. Velasco, Phys. Rev. E 81, 021706 (2010).

[23] M. Dijkstra and R. van Roij, Phys. Rev. Lett. 89, 208303 (2002).

[24] Y. Martínez-Ratón, J. A. Capitán and J. A. Cuesta, Phys. Rev. E 77, 051205 (2008)

[25] J. A. Capitán, Y. Martínez-Ratón and J. A. Cuesta, J. Chem. Phys. 128, 194901 (2008)

[26] This result can be easily obtained from the chain rule $\Gamma=\bar{\rho} \partial \mathcal{F}_{V} / \partial \bar{\rho}-\mathcal{F}_{V}=$ $V^{-1}\left[\bar{\rho} \sum_{i} \int d z\left(\delta \mathcal{F} / \delta \rho_{i}(z)\right)\left(\partial \rho_{i}(z) / \partial \bar{\rho}\right)-\mathcal{F}\right]$, from the definition $\bar{\rho} \partial \rho_{i}(z) / \partial \bar{\rho}=\bar{\rho} x_{i}(z)=\rho_{i}(z)$ and from the equilibrium conditions $\delta \mathcal{F} / \delta \rho_{i}(z)=\mu_{i}$ to be applied when $\rho_{i}(z) \neq 0$, resulting 
in $\Gamma=V^{-1}\left[\sum_{i} \mu_{i} \int d z \rho_{i}(z)-\mathcal{F}\right]=-\Omega / V$.

[27] See http://www.physics.es/confined/binarymixture.html.

[28] R. Evans and U. Marini Bettolo Marconi, J. Chem. Phys. 86, 7138 (1987). 